\title{
Análise de custos e rendimentos de diferentes métodos de corte de cipós para produção de madeira na floresta nacional do Tapajós
}

\author{
Gustavo Stancioli Campos de PINHON, Nilton Cesar FIEDLER², Pompeu Paes GUIMARÃES³, Gilson \\ Fernandes SILVA ${ }^{4}$, Joaquim dos SANTOS 5
}

\begin{abstract}
RESUMO
Esta pesquisa foi realizada na Floresta Nacional do Tapajós, Pará, e foram avaliados os custos e rendimentos de quatro diferentes Métodos de corte de cipós na condução do manejo florestal, visando à produção de madeira em toras. Cada método foi conduzido numa área de 96 ha e a metodologia consistiu em cortar os cipós em torno de todas as árvores comerciais e potencialmente comerciais (Método M1-Individual), apenas na área de ocorrência de árvores comerciais e potencialmente comerciais (Método M2-Zoneado), em torno de todas as árvores comerciais e potencialmente comerciais e na direção de queda (Método M3-direção de queda) e apenas em torno das árvores comerciais destinadas à primeira colheita (Método M4). Em todas as variáveis analisadas os métodos utilizados diferiram estatisticamente. O método M4, com o menor custo (6,35 US\$) e maior rendimento (2,07 ha/hora) é considerado o melhor desta pesquisa. Neste método, a população de cipós é parcialmente preservada, mantendo, assim, sua importante função ecológica na floresta.
\end{abstract}

PALAVRAS-CHAVE: Manejo florestal, Colheita em áreas manejadas, Custos, Corte de cipós.

\section{Cost and income analysis of different methods of cutting vines for wood production in the Tapajós national forest}

\section{ABSTRACT}

This survey was conducted in the Tapajós National Forest, in the state of Pará, Brazil evaluating the cost and income of four different methods of cutting vines in the course of forest management, looking to log wood production. Each method was conducted in an area of 96 ha by cutting the vines (1) around all commercial and potentially commercial trees (Method M1), (2) only in the area of commercial and potentially commercial tree occurrence (Method M2), (3) around al commercial and potentially commercial trees and toward fall (Method M3), and (4) only around those commercial trees chosen for the first harvest (Method M4). In all variables analyzed, the methods differed statistically.Method M4 which showed the lowest cost (6.35 U.S. \$), may be considered the best. In this method, the vine population was partially preserved, which is important ecologically.

KEY WORDS: Forest management, Harvest in management areas, Cost, Vines cutting.

\footnotetext{
${ }_{1}$ IBAMA - Brasilia - DF - SAIN, Av. L4 Norte - CEP 70910-900 Brasília - DF. E-mail: pinhogustavo@hotmail.com

2 Universidade do Espírito Santo - UFES. E-mail: niltoncesarfiedler@yahoo.com.br

3 Universidade Federal do Espírito Santo (UFES).E-mail: pompeupaes@yahoo.com.br

${ }^{4}$ Universidade do Espírito Santo - UFES. E-mail: gfsilva2000@yahoo.com.br

${ }^{5}$ Instituto Nacional de Pesquisas da Amazônia - AM. E-mail: joca@inpa.gov.br
} 


\section{INTRODUÇÃO}

No bioma amazônico, o manejo florestal se constitui, atualmente, como uma técnica de conservação dos ecossistemas que propicia o uso da madeira de forma planejada, melhoria das condições de vida das populações e a manutenção da floresta. Desta forma, o manejo tende a aliar as demandas do setor de forma tecnificada e sustentada.

Nas florestas tropicais, os cipós são característicos de várias formações e são úteis em múltiplas funções no ecossistema tais como alimentação, transpiração, abrigo e deslocamento de fauna. No entanto, segundo Gerwing \& Vidal (2002), os cipós podem também diminuir o crescimento das árvores e aumentar as clareiras na derrubada de árvores selecionadas para abate durante as operações de manejo para produção de madeira.

Para que durante a intervenção na floresta o dano às árvores remanescentes seja minimizado, diversos autores citam a necessidade de corte dos cipós que entrelaçam os troncos ou a copa. Para Putz (1991), o corte de cipós deve ser feito anteriormente à colheita como uma das atividades técnicas do manejo em florestas tropicais. Diversos pesquisadores têm analisado o tempo necessário que deve haver entre esta atividade e o abate das árvores. Para Vidal et al. (1998), quando o corte de cipós é feito um ou dois anos antes da colheita, estes ficam decompostos ou bastante enfraquecidos durante a intervenção. Dessa maneira, o dano que normalmente resultaria da forte ligação dos cipós entre a árvore derrubada e as árvores vizinhas seriam menores.

Segundo Scolforo (1998), os seguintes pontos básicos devem ser considerados na condução do manejo florestal sustentável: inventário pré-corte, em que se procurará marcar as árvores desejáveis para corte, a orientação de queda e a distância mínima da árvore derrubada para a trilha de arraste; corte de cipós feito um ano antes da colheita, para reduzir danos no momento de derrubar e processar a árvore, além de reduzir a competição de copa; e refinamento do povoamento e desbaste para liberação das árvores remanescentes.

Segundo UHL et al. (1997), uma colheita madeireira manejada, com inventário florestal, corte de cipós e planejamento cuidadoso, tem um custo adicional em torno de US\$72/ha. No entanto, as perdas com a utilização pouco eficiente dos equipamentos e o desperdício de madeira das operações não planejadas são maiores que os custos adicionais associados à colheita madeireira planejada. Portanto, os custos adicionais considerados como devidos à adoção do manejo florestal podem ser compensados com o aumento de eficiência da colheita.

De acordo com FFT (1999), o corte de cipós tem como principal objetivo a facilitação da operação de derrubada das árvores comerciais selecionadas para o abate. Esta ação visa não comprometer a sua queda direcionada e reduzir os danos causados às árvores remanescentes, bem como diminuir os riscos de acidentes durante a colheita e, conseqüentemente, aumentar a segurança das equipes de corte (Pereira Junior $e t$ al., 2006).

Esta pesquisa teve como objetivo principal avaliar os custos e os rendimentos de diferentes métodos de corte de cipós na condução do manejo florestal, visando à produção de madeira em toras, na Floresta Nacional do Tapajós.

\section{MATERIAL E MÉTODOS}

\section{DESCRIÇÃO DA ÁREA}

A pesquisa foi realizada na Floresta Nacional do Tapajós no estado do Pará, localizada entre as coordenadas geográficas de $2^{\circ} 45^{\prime}$ e $4^{\circ} 15^{\prime}$ de Latitude Sul e $54^{\circ} 45^{\prime}$ e $55^{\circ} 30^{\prime}$ de Longitude Oeste. A vegetação predominante na área da FLONA é a Floresta Ombrófila Densa com árvores de grande porte, variando de 25 a $50 \mathrm{~m}$ de altura. O clima é chuvoso, com índice pluviométrico superior a $2.300 \mathrm{~mm}$ por ano e temperatura média de 22 e $25^{\circ} \mathrm{C}$.

$\mathrm{Na}$ FLONA, encontra-se em execução pelo Instituto Brasileiro do Meio Ambiente e dos Recursos Naturais Renováveis - IBAMA o Projeto PD 68/89 Rev.1 (F): "Manejo da Floresta Nacional do Tapajós para Produção Sustentada de Madeira Industrial", contando com financiamento da Organização Internacional de Madeiras Tropicais - ITTO (PINHO, 2006). O Projeto está localizado às margens da BR 163 que liga Santarém a Cuiabá, na altura do km 83 e ocupa uma área de 3.222 ha.

A área do Projeto PD 68/89 possui uma baixa densidade na rede de drenagem, sendo efetivamente drenada pelo Igarapé Branco cujas nascentes se localizam na porção oeste da área de colheita, junto às elevaçôes que separam a bacia hidrográfica do Rio Curuá-Una da bacia do Rio Tapajós.

Segundo EMBRAPA (1999), os solos encontrados na área do projeto são Argissolo Vermelho Amarelo distrófico (PVAd), Latossolo Amarelo distrófico típico originário de Gnaisse granítico leucocrático (Lad1), Latossolo Amarelo distrófico típico originário de Sedimento areno-argiloso (Lad2) e Latossolo Amarelo distrófico típico originário de Arenito (Lad3) , contudo, este estudo foi realizado apenas em áreas com o solo Lad1.

\section{DELINEAMENTO EXPERIMENTAL}

Para a realização desta pesquisa, foram escolhidos quatro blocos do Projeto PD 68/89 de 96 ha cada (1200 X 800 m). Foi adotado o delineamento estatístico "Modelo de blocos ao acaso" e para isso cada um dos blocos referidos anteriormente foi dividido em quatro parcelas de $24 \mathrm{ha}(300 \mathrm{X} 800 \mathrm{~m})$. Os quatro métodos de corte de cipós testados, denominados 
M1 (Individual), M2 (Zoneado), M3 (Direção de queda) e M4 (Individual primeira colheita) foram então distribuídos, aleatoriamente, nessas parcelas (Figura 1).

Em cada bloco, o corte de cipós foi realizado simultaneamente com o censo florestal, aproximadamente um ano antes da colheita (outubro de 2000). A equipe responsável pelo corte dos cipós foi à mesma em todos os métodos e repetiçôes e era composta por um técnico e por dois ajudantes de campo.

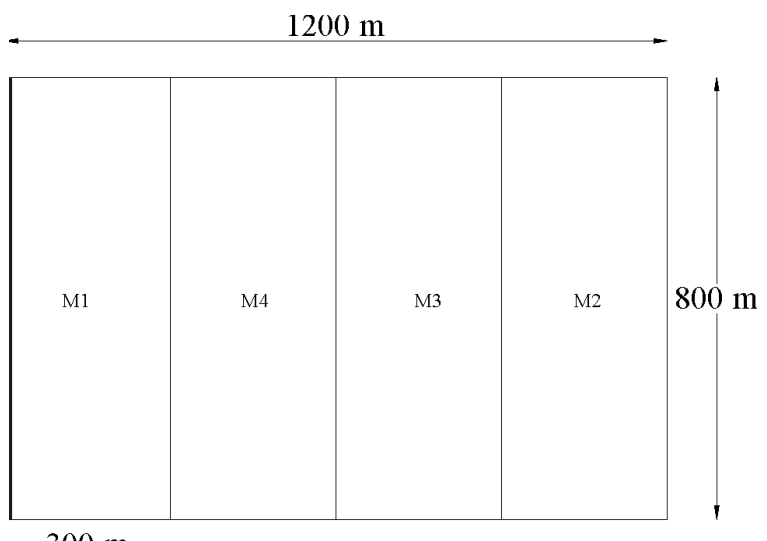

$300 \mathrm{~m}$

Figura 1 - Exemplo do esquema de distribuição dos métodos de corte de cipós dentro dos blocos.

Os métodos de corte de cipós analisados foram os seguintes:

a) Método 1 - M1 (Individual): O corte de cipós foi realizado em torno de todas as árvores comerciais ou potencialmente comerciais, com diâmetro à altura do peito (DAP) igual ou superior a $50 \mathrm{~cm}$. Todos os cipós, com diâmetro acima de $2,5 \mathrm{~cm}$, que se entrelaçavam com a árvore, independentemente da distância do seu enraizamento do tronco da árvore em questão, foram cortados (Figura 2). Foi considerada árvore comercial aquela que possuía madeira com mercado favorável na atualidade e na região prevista para sua comercialização. A árvore potencialmente comercial era aquela que detinha mercado em locais distintos, mas que poderia vir a ser mais valiosa no futuro.

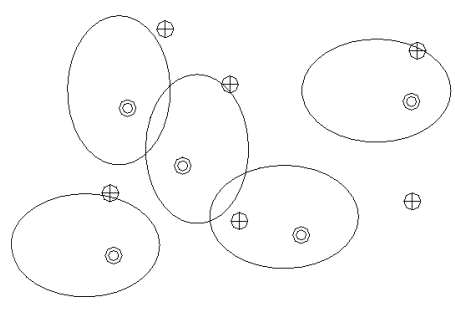

LEGENDA

() Árvore comercial ou potencialmente comercial

$\oplus$ Árvore sem valor comercial

Área do corte dos cipós variável conforme suas ocorrências

Figura 2 - Esquema demonstrativo do corte de cipós do Método 1 - M1 (Individual).

b) Método 2 - M2 (Zoneado): O corte de cipós foi realizado em toda a área de ocorrência de árvores comerciais ou potencialmente comerciais, com DAP igual ou superior a $50 \mathrm{~cm}$. Todos os cipós com diâmetros acima de $2,5 \mathrm{~cm}$ foram cortados, exceto em áreas de não-ocorrência de árvores comerciais ou potencialmente comerciais (Figura 3). Essas áreas tinham, freqüentemente, alta densidade de ocorrência de cipós.

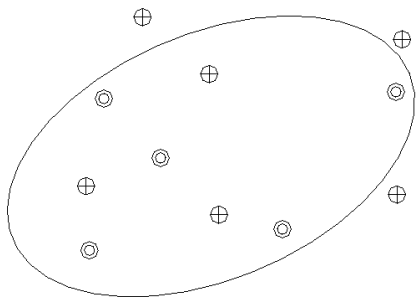

LEGENDA

$$
\begin{aligned}
& \text { Arvore comercial ou } \\
& \text { potencialmente comercial } \\
& \text { Árvore sem valor comercial } \\
& \text { Área do corte dos cipós, } \\
& \text { variável de acordo com a } \\
& \text { ocorrência de árvores } \\
& \text { comerciais, potencialmente } \\
& \text { comerciais e de cipós }
\end{aligned}
$$

Figura 3 - Esquema demonstrativo do corte de cipós do Método 2 - M2 (Zoneado).

c) Método 3 - M3 (Direção de queda): O corte de cipós foi realizado da mesma forma como descrito no Método 1 . Contudo, foi incluído o corte dos cipós presentes na direção estimada de queda das árvores comerciais e potencialmente comerciais considerando a distância máxima para o corte igual à altura da árvore, conforme ilustrado na Figura 4.

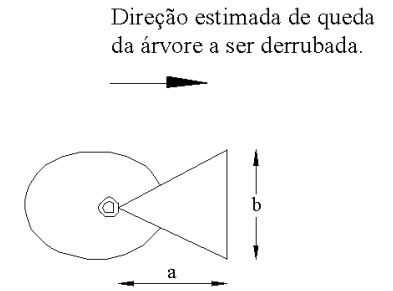

LEGENDA

Árvore a ser derrubada

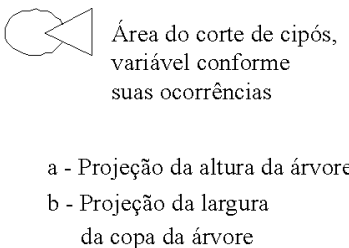

Figura 4 - Esquema demonstrativo do corte de cipós do Método 3 - M3 (Direção de Queda).

d) Método 4-M4 (Individual $1{ }^{\mathrm{a}}$ colheita): Nesse método foi adotado um procedimento idêntico ao descrito no Método 1, limitando o corte de cipós apenas às árvores comerciais selecionadas para derrubada durante a primeira operação de colheita florestal.

As diferenças observadas entre as médias foram comparadas pelo Teste Newman - Keuls, em nível de 1\% de probabilidade (REGAZZI, 1991).

\section{CÁLCULO DOS CUSTOS E RENDIMENTOS}

Os custos de cada método foram avaliados a partir do uso de uma ficha de campo que indicou o rendimento de cada equipe em cada método. Foram registrados nesta ficha, o tempo trabalhado, a área trabalhada (medido com trena) 
e eventuais contratempos ocorridos durante a operação de corte de cipós. Usou-se o método de estudo de tempos contínuos, que consiste em considerar o tempo efetivamente trabalhado.

O rendimento de cada método foi calculado dividindose a área trabalhada, em hectares, pelo tempo efetivamente trabalhado, em horas (ha/h).

Os custos finais de cada método foram obtidos dividindose os custos totais, em dólar, pelos rendimentos de cada método (R\$/ha).

Os cálculos com os custos de cada método foram baseados no rendimento da equipe ao realizar o corte de cipós de acordo com cada método em cada bloco, conforme Tabela 1 .

Tabela 1 - Rendimento da equipe por método de corte de cipós em cada bloco.

\begin{tabular}{lcccc}
\hline & \multicolumn{4}{c}{ Rendimento por método (ha/hora) } \\
\cline { 2 - 5 } Bloco & $\begin{array}{c}\text { M1 } \\
\text { (Individual) }\end{array}$ & $\begin{array}{c}\text { M2 } \\
\text { (Zoneado) }\end{array}$ & $\begin{array}{c}\text { M3 } \\
\text { (Direção da } \\
\text { queda) }\end{array}$ & $\begin{array}{c}\text { M4 } \\
\text { (Individual 1 a } \\
\text { colheita) }\end{array}$ \\
\hline B9 & 1,21 & 1,20 & 1,10 & 2,23 \\
B11 & 1,36 & 1,06 & 1,30 & 2,02 \\
B12 & 1,87 & 0,83 & 1,44 & 2,52 \\
B27 & 1,84 & 1,00 & 1,10 & 1,51 \\
Média & 1,57 & 1,02 & 1,24 & 2,07 \\
\hline
\end{tabular}

A avaliação dos custos baseou-se no custo de pessoal, horas efetivamente trabalhadas, sendo composto por um técnico, com salário de $\mathrm{R} \$ 9.86 /$ hora, e dois ajudantes de campo, com salário de $\mathrm{R} \$ 3,15 /$ hora cada, assim o salário da equipe foi de $\mathrm{R} \$ 16,16 /$ hora; custo do $\mathrm{km}$ rodado do veículo como combustível e manutenção ( $\mathrm{R} \$ 3,97 /$ hora); equipamentos de segurança: bota, capacete. Perneira, etc. (R \$3,41/hora); e material de campo como facão, cantil etc. (R $\$ 0,68 /$ hora); à época (outubro/2000), os custos médios para se efetuar cada método de corte de cipós foram de $\mathrm{R} \$ 24,22 /$ hora, sem considerar os valores residuais dos equipamentos e materiais. Os custos foram calculados em dólar por hora trabalhada $(\mathrm{R} \$ / \mathrm{h})$.

Os valores médios dos custos por hectare foram analisados em dólar, considerando o câmbio de US $\$ 1,00$ equivalente a $R \$$ 1,91, à época do corte de cipós (outubro/2000), sendo obtidos dividindo-se o valor do custo médio por hora ( $\mathrm{R} \$ 12,68 /$ hora $)$ pelo rendimento de cada equipe em cada método e bloco (ha/ hora - Tabela 1), conforme apresentado na Tabela 2.

\section{RESULTADOS E DISCUSSÃO}

\section{COMPARAÇÕES ESTATISTIICAS DOS MÉTODOS}

Os custos apresentados na Tabela 2 foram utilizados para calcular a análise de variância (ANOVA). O resultado da
Tabela 2 - Custos de cada método em cada bloco.

\begin{tabular}{lcccc}
\hline Bloc0 & \multicolumn{4}{c}{ Custo por método (US\$) } \\
\cline { 2 - 5 } & $\begin{array}{c}\text { M1 } \\
\text { (Individual) }\end{array}$ & $\begin{array}{c}\text { M2 } \\
\text { (Zoneado) }\end{array}$ & $\begin{array}{c}\text { M3 } \\
\text { (Direção da } \\
\text { queda) }\end{array}$ & $\begin{array}{c}\text { M4 } \\
\text { (Individual 1 a } \\
\text { colheita) }\end{array}$ \\
\hline B9 & 10,48 & 10,57 & 11,53 & 5,69 \\
B11 & 9,32 & 11,96 & 9,75 & 6,28 \\
B12 & 6,78 & 15,28 & 8,81 & 5,03 \\
B27 & 6,89 & 12,68 & 11,53 & 8,40 \\
Média & 8,37 & 12,62 & 10,40 & 6,35 \\
\hline
\end{tabular}

ANOVA revelou a ocorrência de diferença significativa entre os métodos avaliados, em nível de $5 \%$ de probabilidade pelo teste $\mathrm{F}$. Tendo em vista esta diferença estatística, foi aplicado então o teste de Newman-Keuls para comparar as médias de custo relativas a cada método. Pela observação da Figura 5, pode-se concluir que os métodos Ml, M2 e M3 não apresentaram diferença significativa entre si, da mesma forma que os métodos Ml, M3 e M4.

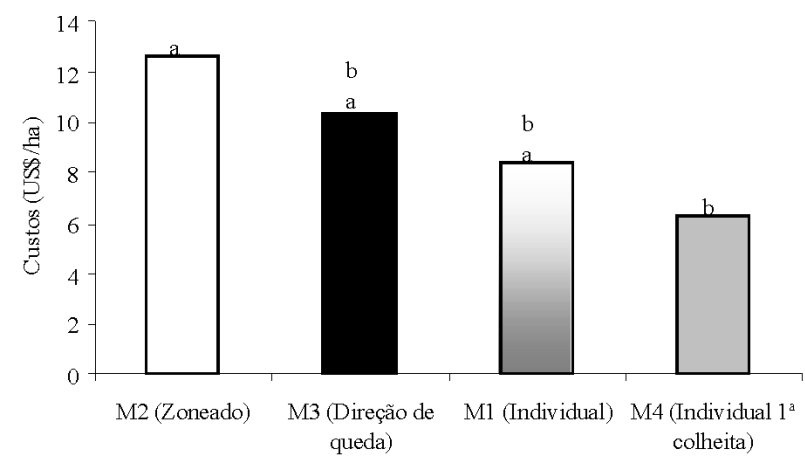

Figura 5 - Gráfico dos custos médios de cada método de corte de cipós indicando o resultado do Teste Newman-Keuls, a $1 \%$ de probabilidade.

Somente os métodos M2 e M4 são diferentes estatisticamente, sendo o primeiro cerca de seis dólares mais caro, em média, que o segundo. A semelhança entre as características dos métodos M1, M3 e M4 explica porque tais métodos possuem os menores custos, ou seja, a equipe do corte de cipós teve de se deslocar menos dentro da mata. No entanto, o método M3, por cortar os cipós relativos ao direcionamento de queda das árvores, mesmo sendo um pouco mais caro dentre esses, favorece a uma maior Segurança dos trabalhadores a diminuição de riscos a acidentes.

O motivo para o método M4 possuir o menor custo (US\$6,35/ha) e o maior rendimento (2,07 ha/hora) baseiase no fato de a sua metodologia exigir o corte de cipós em uma quantidade menor de árvores. Todavia, cortar menor área de cipós indica que, em colheitas subseqüentes, haverá a necessidade de novos cortes de cipós, enquanto que nos outros 
métodos em que o corte de cipó abrange maior área, haverá necessidade de próximos cortes de cipós menos drásticos.

Peto teste de comparação de médias, o método M2 foi o único a apresentar, custo médio maior em relação ao $\mathrm{M} 4$, estatisticamente comprovado em nível de $1 \%$ de probabilidade pelo Teste Newman - Keuls. Este resultado pode ser explicado pelo fato de que no M2 é feito o corte de cipós de uma área maior do que dos outros métodos. Os métodos M1 e M3, embora um pouco mais simples não diferiram dos demais.

O método M4 foi considerado o melhor do ponto de vista da análise de custos, além do fato de a população de cipós ser melhor preservada em relação aos demais métodos de corte analisados, mantendo, assim, sua importante função ecológica na floresta.

\section{CONCLUSÕES}

O menor custo final foi obtido no Método M4 (US\$ 6,35/ ha). Neste método, além de ter havido um maior rendimento (2.07 ha/hora), a população de cipós foi melhor preservada;

Com base nos resultados, podem ser feitas algumas sugestões para futuros estudos: deve-se realizar um levantamento da população de cipós antes de se aplicar o método de corte para avaliar a contribuição da massa foliar desses cipós como componente do Índice de Área Foliar do dossel da floresta em questão e a eficiência do corte; e deve ser maior o número de repetições de cada método.

\section{BILBIOGRAFIA CITADA}

EMBRAPA. 1999. Centro Nacional de Pesquisa de Solos (Rio de Janeiro). Sistema brasileiro de classificação de solos. Brasília: EMBRAPA-SPI. 412pp.
FFT - Fundação Floresta Tropical. 1999. Manual de procedimentos técnicos para condução de manejo florestal e exploração de impacto reduzido (versão 3.1). Belém. 90p.

Gerwing, J.J.; Vidal, E. 2002. Changes in liana abundance and species diversity eight years after liana cutting and logging in an eastern Amazonian forest. Conservation Biology, 16(2): 544-548.

Pereira Júnior, R.A.; Martins, D.P.; Pereira, D.C. 2006. Práticas de manejo e exploração florestal no âmbito do projeto Tapajós. In: Pereira Junior, R. A. (org). Floresta Nacional do Tapajós: experiências e lições para implementação do manejo florestal em Unidades de Conservação. Belém: Projeto Tapajós. p. 35-92.

Pinho, G.S.C. 2006. O Projeto ITTO PD 68/89 - Manejo na Floresta Nacional do Tapajós para a produção sustentada de madeira industrial. In: Pereira Junior, R. A. (org). Floresta Nacional do Tapajós: experiências e lições para implementação do manejo florestal em Unidades de Conservação. Projeto Tapajós. p. 27-34.

Putz, F.E. 1991. Silvicultural effects of lianas. In: F.E. Putz \& H.A. Mooney, (eds.) The Biology of Vines. Cambridge University Press, Cambridge. p. 493-501.

Regazzi, A.J. 1991. Análise de variância e testes de significância. Viçosa: Sociedade de Investigaçõos Florestais - SIF. 111pp.

Scolforo, J .R.S. 1998. Manejo Florestal. Lavras: UFLA/FAEPE. 443pp.

Uhl, C.; Barreto, P.; Veríssimo, A.; Barros, A.C.; Amaral, P.; Vidal, E.; Souza JR, C. 1997. Uma abordagem integrada de pesquisa sobre o manejo dos recursos naturais na Amazônia. Série Amazônica no 7 -IMAZON. Belém. 29pp.

Recebido em 22/08/2008

Aceito em 13/12/2008 
